\title{
Evaluación de las concentraciones de lípidos y apoproteínas A-I y B-100 en un grupo de escolares de cinco departamentos del centro-oriente de Colombia
}

\author{
Elpidia Poveda ${ }^{1}$, Ney Callas ${ }^{1}$, César Baracaldo ${ }^{1}$, Carlina Castillo ${ }^{1}$, Patricia Hernández ${ }^{2}$, \\ Martha Guerra ${ }^{2}$ \\ ${ }^{1}$ Grupo de Nutrición, Subdirección de Investigación, Instituto Nacional de Salud, Bogotá D.C., Colombia \\ ${ }^{2}$ Grupo de Investigación Clínico-Genético-Molecular en Dislipoproteinemias, Departamento de Ciencias, \\ Pontificia Universidad Javeriana, Bogotá D.C., Colombia
}

Introducción. Existe evidencia sobre variaciones en las concentraciones de lípidos y apoproteínas en los niños asociados a cambios fisiológicos o al medio ambiente, este último puede inducir alteraciones futuras en los lípidos.

Objetivo. Evaluar las concentraciones de colesterol total, colesterol LDL, colesterol HDL y apoproteínas A-1 y B-100 en escolares de cinco departamentos del centro-oriente colombiano, y comparar por edad, sexo, índice de masa corporal, práctica de actividad física y preferencias hacia algunos alimentos.

Materiales y métodos Se recolectaron 741 muestras de sangre que se analizaron por métodos enzimáticos, colorimétricos e inmunoturbidimétricos. Todos los valores se presentaron como media y desviación estándar y para la comparación entre grupos se utilizó prueba t, ANOVA y Scheffe.

Resultados. Se observaron variaciones en los lípidos y valores constantes en las apolipoproteínas, las concentraciones de los lípidos se relacionaron con los cambios de edad y sexo. Se observaron cambios que pueden asociarse al grado de maduración sexual, dieta y nivel de actividad física. Los valores de colesterol total, colesterol LDL y colesterol HDL fueron más bajos a los reportados en otros grupos de población mientras que en los triglicéridos hay mayor variación con tendencia a ser superiores.

Conclusión. Existen otras variables diferentes a la edad y el género que influyen en las variaciones de los lípidos; se requieren otros estudios para determinar la contribución de cada factor. La promoción de estilos de vida saludable puede ayudar a disminuir el riesgo futuro de enfermedad cardiovascular asociada a bajas concentraciones de colesterol HDL y mayores de triglicéridos en esta población.

Palabras clave: colesterol, triglicéridos, apolipoproteína A-I, apolipoproteína B-100, niño, adolescente.

Lipid and apoprotein A-I and B-100 levels in school children school from five centraleastern provinces of Colombia

Introduction. Lipid and apolipoprotein are known to vary in children. These variations can be associated to physiologic changes or the environment; this last one probably induces future alterations in the lipids.

Objetive. The levels of total cholesterol, LDL-cholesterol, HDL-cholesterol, and apoproteins AI and B-100 were compared in school children from five provinces of middle-eastern Colombia. Associations were made with age, gender, body mass index, fitness activity, and food preference. Materials and methods. 741 blood samples were collected and biochemical determinations were undertaken with standard enzymatic, colorimetric, and inmunoturbidimetric methods. All values are presented as mean \pm SE, comparisons among groups were made using Student's t test, ANOVA and Scheffe.

Results. Lipid variation was observed in the school children, however in contrast, the concentrations of Apoproteins ApoB-100 and ApoA-I tended to be constant. The lipid fluctuations 
showed some relationship with changes in age and gender, however, changes were also observed that indicate a possible relationship with sexual maturation, diet and fitness activity. An important finding was that the HDL cholesterol value was lower that reported from other countries, while the triglycerides was higher and this may indicate a future increased risk for cardiovascular diseases.

Conclusion. Fluctuations occur in the lipid levels and vary with age and gender. Associations with other factors may be present; however, more research is necessary to determinate the contribution of each factor. A promotion campaign among school-age children for a healthier life style may contribute to a decrease in future risk for cardiovascular diseases associated with low HDL-cholesterol levels and higher triglycerides.

Keywords: cholesterol, triglycerides, apolipoprotein A-I, apolipoprotein B-100, child, adolescent.

El perfil lipídico y las lipoproteínas presentan cambios asociados con el medio ambiente, el sexo, la etnia y diferencias biológicas y metabólicas (1-12). La contribución del ambiente o de los factores biológicos en la variaciones de los lípidos y lipoproteínas parece ser diferente según la variable lipídica; las concentraciones séricas de colesterol total, colesterol HDL y ¿Lp(a)? están más influenciadas por factores genéticos, mientras que las concentraciones de triglicéridos varían principalmente por el efecto ambiental. En los primeros años de vida y en la pubertad, las concentraciones de lípidos sanguíneos evidentemente dependen más del sexo, de indicadores que reflejan la grasa corporal, el estado nutricional y las diferencias biológicas y metabólicas propias de esta etapa de la vida en la que la maduración sexual juega un papel fundamental (5-14).

En algunos estudios, las niñas en edad preescolar han presentado valores más elevados de colesterol total y colesterol LDL que los niños y muestran disminuciones con la pubertad (9). Las concentraciones de triglicéridos, al contrario del colesterol total y del colesterol LDL, aumentan en forma continua con la edad e independientemente del sexo, mientras que los valores de colesterol HDL se mantienen constantes, con cambios muy

\footnotetext{
Correspondencia:

Elpidia Poveda, Grupo de Nutrición, Subdirección de Investigación, Av. Calle 26 № 51-60, zona 6, CAN, Bogotá, D. C., Colombia

Teléfono: 220 7700, extensión 303; fax: 220 7700, extensión 255

epoveda@ins.gov.co o epoveda1@yahoo.com

Recibido: 02/11/06; aceptado: 14/05/07
}

pequeños en las niñas durante la adolescencia y disminuciones con la edad en los varones $(15,16)$. Los estudios atribuyen estos cambios y diferencias entre sexos a las fluctuaciones hormonales y metabólicas que pueden ser más complejas en las niñas que en los niños, debido a las variaciones ocurridas durante el ciclo menstrual $(17,18)$. En algunas investigaciones se ha demostrado correlación entre la apolipoproteína B-100 (Apo B-100) y el colesterol LDL en ambos sexos; además, incrementos de la Apo B-100 al aumentar la edad. Por el contrario, parece existir poca relación entre apolipoproteína A-I (Apo A-I) y colesterol HDL, valor constante de la Apo A-I en niños e incrementos con la edad en las niñas (19).

En una revisión sobre la literatura científica en la cual se analizan las concentraciones de colesterol total en niños, entre los años 1975 y 1996 en 26 países del mundo, se observó una diferencia de $76 \mathrm{mg} / \mathrm{dl}$ al comparar niños de origen europeo con niños africanos. La curva global de colesterol en la población mostró un pico máximo en el preadolescente con valores absolutos casi coincidentes entre los niños y niñas de 3 a 12 años. Después de los 12 años, los valores para los niños disminuyeron hasta los 16 años, mientras que los valores de las niñas se inclinaron al aumento a través de este rango de edad. La curva en la adolescencia tardía demostró que las mujeres tenían valores absolutos persistentemente mayores que los hombres. En el estudio se observó una gran variación en los niveles de colesterol entre las diferentes poblaciones según edad, sexo y raza e, incluso, en las mismas poblaciones durante un período, aunque se observó un modelo aparentemente universal de aumento 
temprano, descenso y subsiguiente incremento en los valores medios de colesterol desde la preadolescencia hasta la adolescencia tardía (20).

Las diferencias en los hallazgos sobre las variaciones de colesterol total y fracciones lipídicas según las distintas variables biológicas de edad, sexo, raza, nivel de maduración sexual u otras variables de tipo ambiental, indican que aún no existe total claridad sobre los niveles de lípidos y sus variaciones desde el punto de vista fisiológico o patológico, lo cual tiene repercusiones con respecto a la definición de medidas de intervención para dislipidemias entre niños y adolescentes y pautas de prevención de la enfermedad cardiovascular en el adulto.

Los estudios sobre la relación entre lípidos circulantes y formación de placa ateromatosa dan mayor importancia como agente aterogénico al incremento en las concentraciones de colesterol LDL $(21,22)$. El estudio de Webber demostró que, aproximadamente, $50 \%$ de los niños que se encuentran por encima del percentil 75 de concentraciones de colesterol total y colesterol LDL y $42 \%$ de los niños en el cuartil más bajo de colesterol HDL, se mantienen en estos mismos rangos de concentración 12 años después de la toma de la muestra inicial (16). De comprobarse esta tendencia, sería factible la identificación temprana del aumento de los valores de colesterol LDL en niños para diseñar estrategias dirigidas a disminuir el riesgo de desarrollar enfermedad cardiovascular.

El Instituto Nacional de Salud (INS) llevó a cabo una investigación en escolares, con el objetivo de estudiar los factores que determinan la situación nutricional, estado de salud y algunos aspectos socioculturales de los escolares colombianos entre 5 y 15 años que asisten a instituciones educativas públicas y privadas, el cual no se culminó por recursos insuficientes. De esta investigación surgió el interés por evaluar en los niños variables conocidas como factores de riesgo para enfermedades cardiovasculares, las cuales corresponden a una de las principales causas de morbimortalidad en Colombia $(23,24)$. La mayoría de los estudios referentes a factores de riesgo cardiovascular en Colombia se han adelantado en población adulta y se conoce muy poco de lo que desde la niñez podría llegar a aumentar la predisposición futura para este tipo de enfermedades; se sabe que los niños están expuestos a eventos adversos que se pueden controlar para reducir el riesgo cardiovascular futuro del adulto y, así, disminuir la demanda por servicios de salud $(25,26)$. A partir de esta inquietud, se elaboró un nuevo proyecto titulado "Caracterización de algunos factores de tipo ambiental y metabólico de riesgo cardiovascular en la población escolar de la región oriental, Colombia".

En esta publicación se reportan los resultados de las concentraciones medias de lípidos y apolipoproteínas A-I y B-100 en una muestra de escolares de cinco departamentos del centrooriente colombiano y se relacionan con edad y sexo. El estudio se efectuó en estos departamentos considerando que en ellos se presentaban altas tasas de mortalidad por este tipo de enfermedades. Según el archivo de defunciones del Departamento Administrativo Nacional de Estadística, para 1999 la menor tasa de mortalidad por 100.000 habitantes para enfermedad cardiovascular por departamento se presentó en el Vaupés, con un 20,5, y la mayor en Caldas, con una tasa de 176,2; para Santander la tasa fue de 163,4, en Boyacá, de 156,6, en Cundinamarca, de 133,6, en Meta, de 120,3 y en Norte de Santander, de 119,9 (23,24).

El aporte de estos resultados es de tipo descriptivo sobre las variaciones en las concentraciones de lípidos y apolipoproteínas en este grupo de población y su relación con variables de edad, sexo y algunas que indican práctica de actividad física, dimensión corporal y preferencia por el consumo de un grupo específico de alimentos. Estos resultados enriquecen el conocimiento sobre el comportamiento de estas variables en poblaciones colombianas y permiten discurrir sobre la similitud o diferencia con otras poblaciones de escolares. El conocimiento de los niveles de lípidos en la población joven contribuye, además, a orientar las políticas de prevención de la enfermedad cardiovascular, las cuales son de trascendental importancia para disminuir la morbilidad y mortalidad en el adulto por enfermedades crónicas no transmisibles. 


\section{Materiales y métodos}

El estudio es descriptivo analítico transversal y se llevó a cabo en 741 escolares de colegios públicos y privados de los departamentos de Boyacá, Cundinamarca, Meta, Santander y Norte de Santander. La muestra corresponde a 47,4\% de los 1.567 escolares seleccionados en el estudio del INS en el cual se pretendía estudiar los factores que determinan la situación nutricional, estado de salud y algunos aspectos socioculturales de los escolares colombianos entre 5 y 15 años que asisten a instituciones educativas públicas y privadas.

La muestra de 1.567 escolares elegida para la región del centro-oriente colombiano era representativa, aleatoria y estratificada (urbana y rural) de los escolares que asisten a colegios públicos y privados de los cinco departamentos. La selección se basó en la población escolar matriculada en las instituciones públicas y privadas de la región, teniendo en cuenta sexo, edad, tipo de institución y zona. En cada departamento los municipios se seleccionaron de forma aleatoria y aquéllos que no aceptaron participar en el estudio se reemplazaron por otros que admitieron su ingreso. En la selección de los colegios se tuvo en cuenta la lista de colegios públicos y privados provista por las secretarías de educación municipales. Los colegios que no aceptaron participar se reemplazaron por otros que admitieron su ingreso al estudio. Se excluyeron de la muestra los colegios que no tenían el número de niños necesario por curso.

Bacteriólogas del Grupo de Nutrición del INS recolectaron las muestras de sangre en tubos al vacío y sin anticoagulante; la muestra se tomó con el niño en ayuno previo de 12 horas. Los sueros se separaron por centrifugación a 3.000 rpm dentro de las tres horas después de la toma de las muestras y se mantuvieron refrigerados durante este tiempo y el de transporte al INS. Posteriormente, se procesaron manteniéndolos a $-70^{\circ} \mathrm{C}$ mientras se analizaban.

Luego de la recepción de los escolares y la toma de muestras de sangre, las nutricionistas dietistas interrogaron al escolar sobre actividad física y preferencia de consumo de algunos alimentos. El interrogatorio sobre preferencia de consumo de alimentos se realizó únicamente en los niños mayores de siete años, teniendo en cuenta los resultados de la fase de estandarización y validación de procedimientos. Para la evaluación de los parámetros antropométricos de peso y estatura, se utilizaron balanzas electrónicas con sensibilidad de 100 gramos, marca Tanita y tallímetros de madera con una escala en milímetros y centímetros. El índice de masa corporal se clasificó por percentiles de acuerdo con las siguientes categorías: bajo peso $<\mathrm{p} 15$; normal $\mathrm{p} 15<\mathrm{p} 85$; sobrepeso $\mathrm{p} 85<\mathrm{p} 95$; obesidad p95 (27-29).

Las concentraciones de colesterol total y triglicéridos se determinaron utilizando las metodologías enzimáticas-calorimétricas de Serapak-Bayer. El colesterol HDL se determinó en el sobrenadante después de centrifugación y tras la precipitación del colesterol LDL con ácido fosfotúngstico e iones de magnesio. Las concentraciones de Apo-Al y ApoB-100 se determinaron por inmunoturbidimetría (SerapakBayer). Las concentraciones de colesterol LDL se calcularon con la formula de Friedewald cuando las muestras no tuvieron triglicéridos mayores de $200 \mathrm{mg} / \mathrm{dl}$ (30). Las determinaciones se realizaron en el autoanalizador Selectra II Vitalab (Merck). Los procedimientos para toma de muestra y determinaciones bioquímicas fueron previamente validados y estandarizados.

\section{Análisis estadístico}

Se definieron cinco rangos de edad (5-6, 7-8, 9$10,11-12$ y 13-15) con el interés de tener un mayor rango de observación sobre la posible variabilidad en las concentraciones de lípidos y apolipoproteínas en los escolares. En cada rango de edad, sexo y clasificación de índice de masa corporal, se calcularon los percentiles y los valores medios de las concentraciones de colesterol total, triglicéridos, colesterol HDL, colesterol LDL, ApoA-I y ApoB-100. Se efectuaron comparaciones por el análisis de varianzas (ANOVA) y cuando se encontró significancia estadística se realizó el test de comparaciones múltiples de Scheffe. La comparación entre sexos, práctica de actividad física y preferencia por el consumo de algunos 
alimentos, se realizó a través del estadístico t; antes de aplicar la prueba t, se verificó la homogeneidad de las varianzas por la prueba de Levene $(p>0,05)$. En todos los análisis se utilizó el programa estadístico SPSS, manejando un nivel de confianza del $95 \%$.

\section{Consideraciones éticas}

La participación de los colegios y de los niños en el estudio fue voluntaria. El ingreso de los niños fue previamente autorizado por el acudiente del niño mediante la firma del consentimiento informado. La investigación fue aprobada por el Comité de Ética del INS y el Comité de Investigaciones de la Pontificia Universidad Javeriana, siguiendo los lineamientos de la resolución № 008430 de 1993 del Ministerio de Salud de Colombia y en concordancia con la declaración de Helsinki.

\section{Resultados}

En el cuadro 1 se describe la distribución de la muestra. Debido a que no fue posible obtener suficiente cantidad de sangre de algunos niños, 64 muestras no fueron procesadas para colesterol total, colesterol HDL, triglicéridos y colesterol LDL y, 41, para apoproteínas.

En el cuadro 2 se presentan las concentraciones de los lípidos y las apoproteínas hallados en la población estudiada, según sexo e intervalos de edad. Se encontraron valores medios de concentraciones de colesterol total, colesterol LDL, triglicéridos y ApoB-100 más altos en las niñas que en los niños, y concentraciones de colesterol HDL y ApoA-I mayores en los niños. Teniendo en cuenta el grupo de edad y el sexo, se observó la misma tendencia, con diferencias significativas para colesterol total y colesterol LDL entre las niñas y niños de 5-6 años, 7-8 años y 13-15 años y sin diferencias significativas en las categorías de 9 a 12 años. Para colesterol HDL y Apo B-100, las diferencias sólo fueron estadísticamente significativas entre los niños y niñas de 9-10 años y, para Apo B-100, entre los de 13-15 años (cuadro 2).

En los niños, el colesterol total y el colesterol LDL aumentan progresivamente hasta la edad de 9-10 años; posteriormente, disminuyen hasta alcanzar la concentración más baja a los 13-15 años. Este valor es significativamente diferente con los grupos de edad de 7 a 12. El colesterol HDL es mayor en el grupo de 5-6 años y es más bajo a los 13-15 años, pero no se observaron diferencias significativas entre grupos. Los triglicéridos aumentaron progresivamente hasta los 11-12 años, para luego disminuir en el grupo de 13-15 años; se encontraron diferencias estadísticamente significativas entre el grupo de 5-6 y el de 11-12 años. Las Apo A-I y B-100 no mostraron diferencias significativas entre los grupos de edad; no obstante, se observó la concentración más baja en el grupo de 13-15 años (cuadro 2).

En las niñas, el colesterol total y el colesterol LDL aumentaron hasta los 7-8 años y disminuyeron a partir de los 9-10 años, alcanzando la concentración más baja a los 13-15 años; las diferencias para colesterol total fueron estadísticamente significativas entre los grupos de 7-8 años y 13-15 años y, para colesterol LDL, entre los 7-8 años y los grupos de 11 a 15 años (cuadro 2). Los triglicéridos aumentaron

Cuadro 1. Distribución de la muestra según sexo, edad y tipo de colegio. Escolares de cinco departamentos del centrooriente colombiano.

\begin{tabular}{|c|c|c|c|c|c|c|c|c|c|c|}
\hline \multirow[t]{3}{*}{ Edad (años) } & \multicolumn{2}{|c|}{ Masculino } & \multicolumn{2}{|c|}{ Femenino } & \multicolumn{4}{|c|}{ Tipo de colegio } & \multicolumn{2}{|c|}{ Total } \\
\hline & \multirow[t]{2}{*}{$\mathbf{n}$} & \multirow[t]{2}{*}{$\%$} & \multirow[t]{2}{*}{$\mathbf{n}$} & \multirow[t]{2}{*}{$\%$} & \multicolumn{2}{|c|}{ Oficial } & \multicolumn{2}{|c|}{ Privado } & \multirow[t]{2}{*}{$\mathbf{n}$} & \multirow[t]{2}{*}{$\%$} \\
\hline & & & & & $\mathbf{n}$ & $\%$ & $\mathbf{n}$ & $\%$ & & \\
\hline $5-6$ & 57 & 7,7 & 54 & 7,3 & 73 & 9,8 & 38 & 5,1 & 111 & 15,0 \\
\hline $7-8$ & 71 & 9,6 & 89 & 12,0 & 78 & 10,5 & 82 & 11,1 & 160 & 21,5 \\
\hline $9-10$ & 53 & 7,1 & 64 & 8,6 & 47 & 6,3 & 70 & 9,4 & 117 & 15,8 \\
\hline $11-12$ & 88 & 11,9 & 88 & 11,9 & 108 & 14,6 & 68 & 9,2 & 176 & 23,7 \\
\hline $13-15$ & 82 & 11,1 & 96 & 12,9 & 135 & 18,2 & 43 & 5,8 & 178 & 24,0 \\
\hline Total & 351 & 47,3 & 391 & 52,6 & 441 & 59,4 & 301 & 40,6 & 742 & 100 \\
\hline
\end{tabular}


Cuadro 2. Perfil lípídico y apoproteínas en niños y niñas según el género y grupos de edad, grupo de escolares de cinco departamentos del centro-oriente de Colombia.

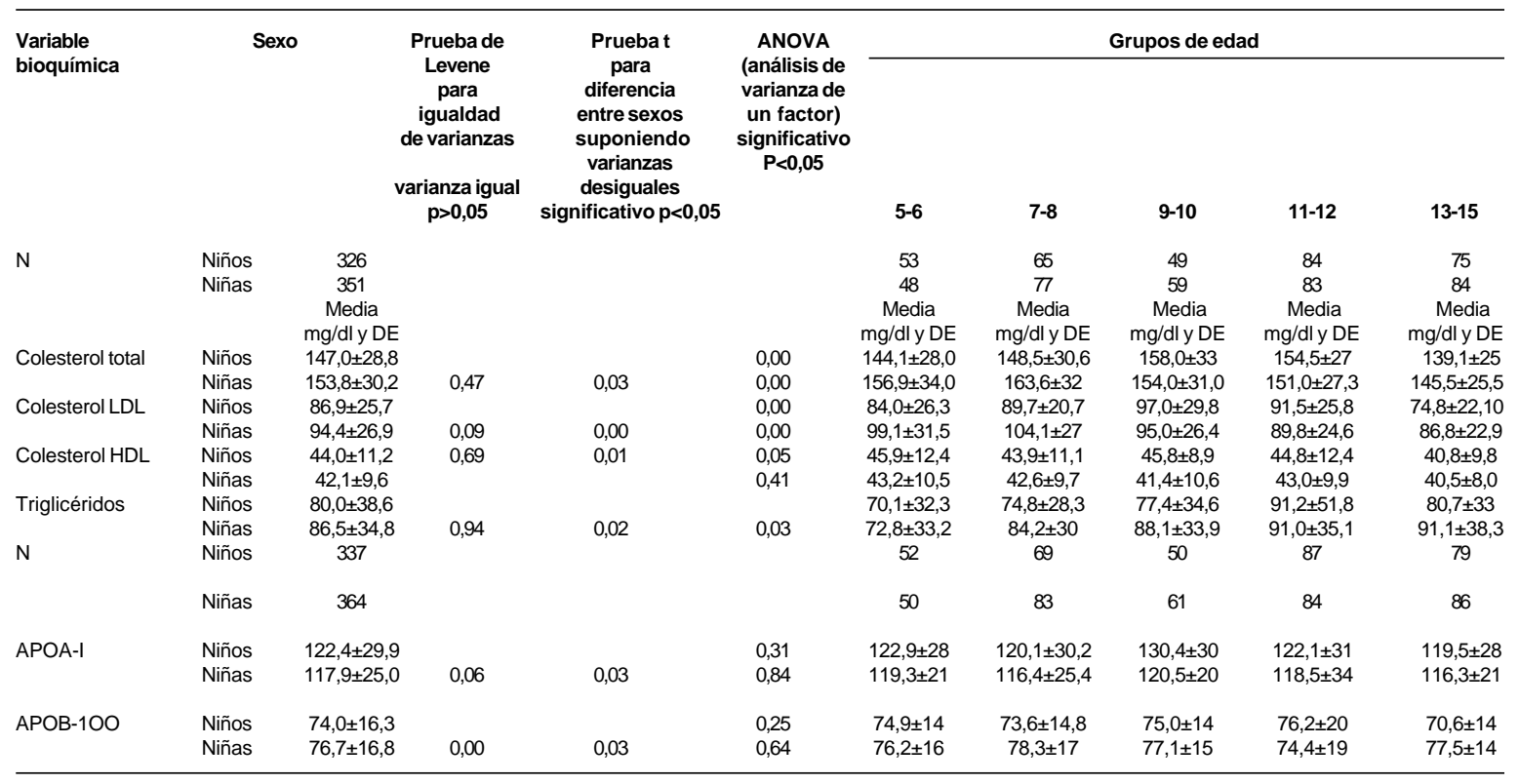

Varianza igual $p>0,05$

Prueba t para diferencia entre sexos suponiendo varianzas desiguales significativo $\mathrm{p}<0,05$

ANOVA (análisis de varianza de un factor) significativo $\mathrm{P}<0,05$-Prueba tpara diferencia entre sexos según grupos de edad y suponiendo varianzas desiguales, significativo $\mathrm{p}<0,05$ :

Colesterol total niñas $V s$. niños de 5-6 años ( $\mathrm{p}=0,043)$; niñas $V s$. niños de 7-8 años $(\mathrm{p}=0,03)$; niños $V s$. niñas de $13-15$ años $(\mathrm{p}=0,001)$. Colesterol LDL niñas $V s$. niños $5-6$ años $(p=0,010)$; niñas $V s$. niños 7-8 años $(0,010)$; niños Vs. niñas de 13-15 años $(p=0,001)$. Colesterol HDL niños Vs. niñas de 9-10 años ( $p=0,022)$ ApoA-I niños $V s$. niñas $9-10$ años $(p=0,044)$ ApoB-100 niños $V s$. niñas de 13-15 años $(p=0,003)$

-Categorías de edad en las que existen concentraciones medias significativamente diferentes en niños y niñas

Según Scheffe las diferencias en cada una de las variables lipídicas se ubican sólo entre las siguientes categorías de edad en niños:

Colesterol total: $13-15$ Vs. 7-8 $(\mathrm{p}=0,013) ; 13-15$ Vs. 9-10 $(\mathrm{p}=0,000) ; 13-15$ Vs. 11-12 $(\mathrm{p}=0,000)$.

Colesterol LDL: $13-15$ Vs. 7-8 $(p=0,015) ; 13-15$ Vs. 9-10 $(p=0,000) ; 13-15$ Vs. 11-12 $(p=0,002)$.

Triglicéridos: 5-6 Vs. 11-12 ( $\mathrm{p}=0,044)$.

Según Scheffe las diferencias en cada una de las variables lipídicas se ubican solo entre las siguientes categorías de edad en niñas:

Colesterol total: 7-8 Vs. 13-15 $(\mathrm{p}=0,006)$

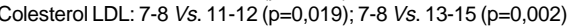

paulatinamente con la edad y el colesterol HDL mostró una tendencia hacia la disminución; sin embargo, no se presentaron diferencias significativas entre grupos. Las apoproteínas tampoco presentaron diferencias significativas entre grupos (cuadro 2).

En el cuadro 3 se observan las concentraciones de los diferentes parámetros estudiados únicamente por intervalos de edad. El colesterol total y el colesterol LDL aumentan hasta llegar a un valor máximo en el grupo de siete años y, posteriormente, van disminuyendo hasta alcanzar el menor valor en los escolares de 13-15 años; el valor encontrado en este grupo es significa- tivamente menor a los valores de los grupos de edad de 7-12 años.

Las concentraciones de colesterol HDL se mantienen estables entre los 7 y los 12 años y disminuyen en el grupo de 13-15 años pero no en forma estadísticamente significativa. En cuanto a los triglicéridos, se encontró que las concentraciones más bajas se presentan en el grupo de 5-6 años, aumentan hasta los 11-12 años y disminuyen nuevamente en el grupo de 13-15 años; las diferencias en las concentraciones son significativas entre el grupo de 5-6 años y los grupos de 11-12 y 13-15 años. Las Apo A-I y B100 no muestran diferencias significativas entre 
Cuadro 3. Concentración de lípidos y apolipoproteínas por grupos de edad en escolares de cinco departamentos del centro-oriente de Colombia.

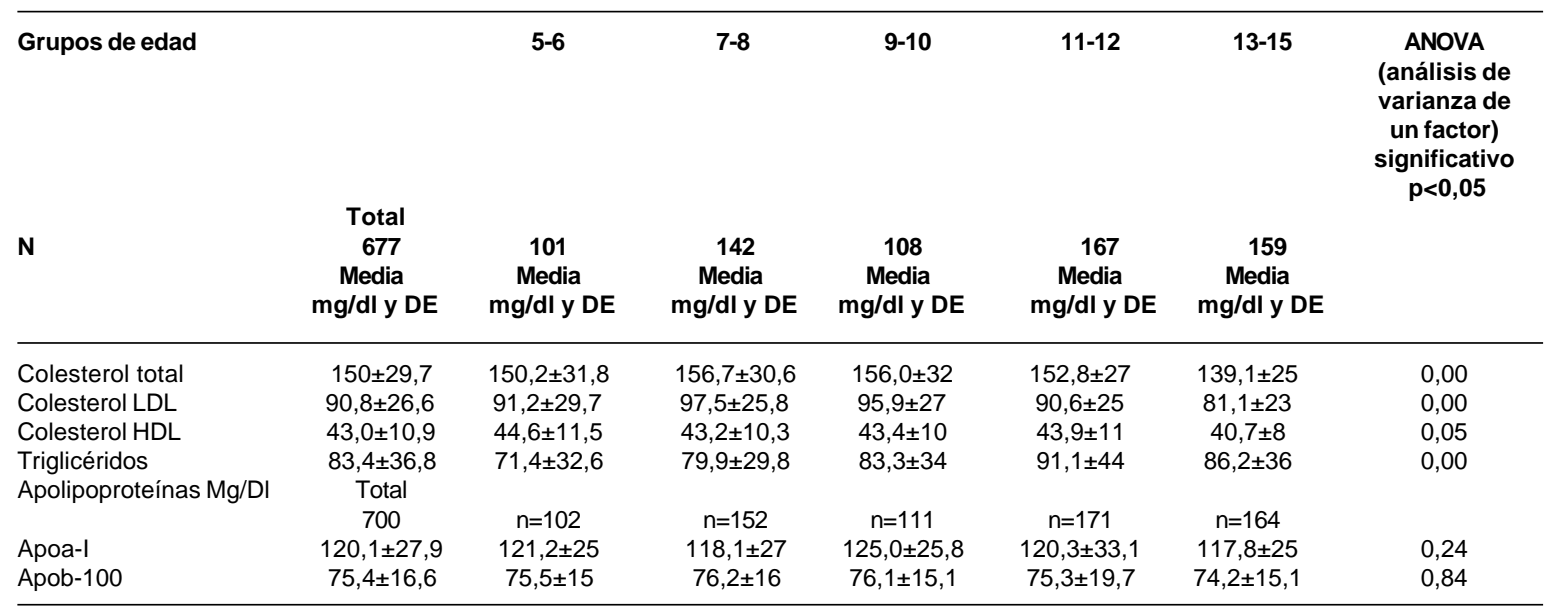

Según Scheffe las diferencias en cada una de las variables lipídicas se ubican entre las siguientes categorías de edad: Colesterol total: 13-15 Vs. 7-8 $(p=0,000)$ 13-15 Vs. 9-10 $(p=0,000) ; 13-15$ Vs. 11-12 $(p=0,001)$.

Colesterol LDL: $13-15$ Vs. 7-8 $(\mathrm{p}=0,000)$; 13-15 Vs. $9-10(0,000)$; 13-15 Vs.11-12 $(\mathrm{p}=0,029)$.

Triglicéridos: 5-6 Vs. 11-12 ( $\mathrm{p}=0,001) ; 5-6$ Vs. 13-15 $(\mathrm{p}=0,038)$

los grupos de edad, aun cuando parecen mantener la tendencia que se encontró para colesterol LDL y colesterol HDL (cuadro 3).

En el cuadro 4 se presentan las concentraciones de lípidos y apoproteínas por percentiles según el sexo y el grupo de edad. Las mayores concentraciones de colesterol total, colesterol LDL, triglicéridos y ApoB-100 se presentaron en las niñas y, de colesterol HDL y ApoA-I, en los niños. En colesterol total y colesterol HDL el percentil 50 (P50) es similar a la media y en colesterol LDL y triglicéridos existen diferencias. Para colesterol LDL la concentración media es mayor que el P50 en los dos sexos y en todos los grupos de edad, excepto en los niños de 5-6, 7-8 y 13-15; en triglicéridos el P50 es menor a la concentración media por edad y sexo, lo cual indica que la distribución de esta variable tiende hacia el lado izquierdo.

El análisis de las concentraciones promedio de cada una de las variables lipídicas, según si los escolares practican o no actividades físicas y según si prefieren consumir algunos alimentos fuente de grasa y azúcares simples, no mostró diferencias estadísticamente significativas, es decir, los valores de lípidos se conservaron iguales entre los escolares, independientemente de la práctica de actividad física o la preferencia de consumo por ciertos alimentos (cuadro 5).

La comparación de las concentraciones medias de lípidos según la clasificación nutricional de índice de masa corporal no demostró diferencias significativas, excepto en los triglicéridos: los escolares que presentaron bajo peso tenían concentraciones más bajas que los escolares con sobrepeso; las demás categorías de índice de masa corporal no mostraron concentraciones de triglicéridos significativamente diferentes (cuadro 6).

\section{Discusión}

Al analizar el perfil lipídico según el sexo pero sin efecto de la edad, se observó que las concentraciones medias de colesterol total, colesterol LDL, triglicéridos eran más elevadas en las niñas que en los niños. Esta misma tendencia se encontró en otros dos estudios realizados en población colombiana $(17,31)$, con valores similares de triglicéridos pero con concentraciones de colesterol total y colesterol LDL más bajas en la población de estudio.

El estudio de parámetros lipídicos de escolares de la ciudad de Medellín mostró un valor medio de colesterol total de $163,3 \pm 29,3 \mathrm{mg} / \mathrm{dl}$ en los 
Cuadro 4. Concentraciones de lípidos y apolipoproteínas según percentiles por grupos de edad y sexo. Grupo de escolares de cinco departamentos del centro-oriente de Colombia.

\begin{tabular}{|c|c|c|c|c|c|c|c|c|c|c|c|c|c|c|c|}
\hline \multirow{2}{*}{$\begin{array}{l}\text { Lípidos y } \\
\text { apoproteínas }\end{array}$} & \multirow{2}{*}{$\begin{array}{c}\text { Grupo de } \\
\text { edad }\end{array}$} & \multicolumn{7}{|c|}{ Niños mg/dl } & \multicolumn{7}{|c|}{ Niñas mg/dl } \\
\hline & & P5 & P10 & P25 & P50 & P75 & P90 & P95 & P5 & P10 & P25 & P50 & P75 & P90 & P95 \\
\hline \multirow[t]{5}{*}{ Colesterol total } & $5-6$ & 96,9 & 109,2 & 123,5 & 145 & 164,5 & 183,8 & 192,1 & 104,3 & 115,9 & 129,5 & 156,0 & 183,7 & 200,0 & 207,9 \\
\hline & $7-8$ & 106,4 & 116,0 & 128,0 & 149,0 & 163,5 & 182,4 & 196,1 & 107,8 & 123,0 & 142,5 & 159,0 & 189,0 & 208,8 & 222,1 \\
\hline & $9-10$ & 107,0 & 118,0 & 139,5 & 157,0 & 179,0 & 191,0 & 205,0 & 100,0 & 113,0 & 133,0 & 153,0 & 170,0 & 194,0 & 210,0 \\
\hline & $11-12$ & 114,0 & 122,0 & 136,5 & 151,0 & 170,7 & 185,5 & 192,0 & 105,6 & 117,0 & 129,0 & 152,0 & 170,0 & 186,0 & 193,2 \\
\hline & $13-15$ & 94,2 & 102,0 & 116,0 & 129,0 & 149,0 & 160,6 & 178,6 & 102,2 & 108,0 & 129,2 & 146,5 & 159,7 & 176,5 & 191,7 \\
\hline \multirow[t]{5}{*}{ Colesterol LDL } & 5-6 & 38,0 & 45,4 & 68,5 & 85,0 & 104,5 & 118,6 & 123,6 & 50,4 & 60,8 & 78,0 & 94,0 & 121,2 & 136,3 & 143,7 \\
\hline & $7-8$ & 55,5 & 61,8 & 74,5 & 91,0 & 102,0 & 123,4 & 125,7 & 59,6 & 69,6 & 87,5 & 100,0 & 127,5 & 144,0 & 156,2 \\
\hline & $9-10$ & 51,0 & 63,0 & 78,0 & 96,0 & 117,0 & 128,0 & 135,5 & 53,0 & 61,0 & 81,0 & 91,0 & 109,0 & 130,0 & 148,0 \\
\hline & $11-12$ & 54,0 & 64,0 & 76,0 & 90,0 & 104,0 & 120,0 & 136,5 & 46,4 & 60,4 & 71,0 & 87,0 & 106,0 & 125,6 & 133,6 \\
\hline & $13-15$ & 36,4 & 44,6 & 62,0 & 72,0 & 89,0 & 102,4 & 113,2 & 52,2 & 56,0 & 70,0 & 87,5 & 98,5 & 115,5 & 129,2 \\
\hline \multirow{5}{*}{ Colesterol HDL } & $5-6$ & 26,4 & 31,0 & 37,5 & 45,0 & 53,0 & 58,6 & 65,9 & 23,8 & 29,7 & 35,5 & 42,5 & 52,0 & 56,1 & 59,1 \\
\hline & $7-8$ & 26,3 & 31,6 & 37,0 & 43,0 & 49,5 & 61,2 & 67,1 & 28,0 & 29,8 & 35,0 & 41,0 & 49,0 & 57,2 & 63,1 \\
\hline & $9-10$ & 31,5 & 34,0 & 39,5 & 46,0 & 52,5 & 58,0 & 60,0 & 22,0 & 29,0 & 35,0 & 41,0 & 48,0 & 56,0 & 60,0 \\
\hline & $11-12$ & 25,0 & 29,0 & 35,0 & 43,5 & 53,7 & 61,5 & 68,5 & 28,2 & 30,0 & 34,0 & 42,0 & 50,0 & 56,6 & 60,0 \\
\hline & $13-15$ & 27,0 & 28,6 & 33,0 & 39,0 & 49,0 & 54,6 & 61,2 & 29,0 & 30,0 & 35,0 & 40,0 & 46,0 & 51,5 & 54,0 \\
\hline \multirow[t]{5}{*}{ Triglicéridos } & 5-6 & 32,1 & 37,8 & 49,5 & 62,0 & 86,0 & 117,2 & 145,2 & 36,1 & 41,9 & 46,5 & 63,5 & 90,0 & 116,6 & 154,2 \\
\hline & $7-8$ & 33,3 & 37,0 & 53,5 & 72,0 & 90,5 & 113,6 & 136,1 & 43,9 & 47,0 & 60,5 & 81,0 & 106,5 & 117,2 & 140,4 \\
\hline & $9-10$ & 33,0 & 38,0 & 50,5 & 77,0 & 95,0 & 120,0 & 140,5 & 49,0 & 50,0 & 63,0 & 78,0 & 109,0 & 129,0 & 174,0 \\
\hline & $11-12$ & 39,0 & 42,5 & 53,0 & 76,5 & 110,5 & 172,0 & 200 & 48,2 & 52,0 & 64,0 & 85,0 & 111,0 & 129,6 & 165,2 \\
\hline & $13-15$ & 34,0 & 45,0 & 54,0 & 74,0 & 95,0 & 136,4 & 150,2 & 39,2 & 51,0 & 66,2 & 80,5 & 113,2 & 147,5 & 171,0 \\
\hline \multirow[t]{5}{*}{ APOA-I } & $5-6$ & 71,2 & 88,2 & 106,4 & 120,5 & 137,8 & 166,9 & 181,9 & 82,2 & 87,3 & 106,9 & 121,0 & 130,3 & 149,0 & 157,1 \\
\hline & $7-8$ & 65,0 & 89,0 & 103,5 & 121,0 & 139,4 & 156,0 & 171,0 & 74,4 & 83,6 & 103,0 & 120,0 & 135,0 & 147,1 & 151,8 \\
\hline & $9-10$ & 85,2 & 90,1 & 115,0 & 124,5 & 142,3 & 177,9 & 192,7 & 92,2 & 96,7 & 106,0 & 117,0 & 131,7 & 148,5 & 169,8 \\
\hline & $11-12$ & 72,5 & 88,9 & 104,0 & 120,0 & 138,0 & 159,6 & 180,6 & 57,7 & 79,9 & 98,0 & 113,7 & 131,7 & 164,3 & 187,5 \\
\hline & $13-15$ & 81,0 & 87,0 & 101,0 & 115,7 & 132,0 & 158,0 & 186,0 & 75,2 & 89,0 & 106,6 & 114,5 & 129,0 & 146,3 & 156,2 \\
\hline \multirow[t]{5}{*}{ APOB-100 } & $5-6$ & 49,8 & 57,8 & 67,1 & 73,3 & 82,6 & 94,5 & 100,4 & 51,2 & 58,4 & 67,7 & 75,5 & 87,1 & 93,9 & 98,9 \\
\hline & $7-8$ & 52,0 & 56,0 & 61,1 & 72,0 & 83,8 & 97,0 & 103,2 & 49,6 & 59,0 & 66,1 & 78,0 & 89,0 & 100,1 & 103,5 \\
\hline & $9-10$ & 49,5 & 55,1 & 63,5 & 74,8 & 86,2 & 95,9 & 99,1 & 56,0 & 59,2 & 65,0 & 76,0 & 87,8 & 99,0 & 106,7 \\
\hline & $11-12$ & 49,6 & 56,9 & 64,0 & 73.0 & 86,0 & 96,8 & 113,8 & 37,9 & 51,8 & 63,6 & 74,0 & 87,0 & 97,0 & 108,5 \\
\hline & $13-15$ & 44,0 & 51,0 & 60,0 & 71,2 & 80,0 & 86,5 & 98,4 & 56,1 & 59,0 & 67,0 & 76,1 & 88,2 & 97,1 & 104,6 \\
\hline
\end{tabular}

niños y de $170,9 \pm 30,1 \mathrm{mg} / \mathrm{dl}$ en las niñas; de colesterol LDL de $102,2 \pm 26,1 \mathrm{mg} / \mathrm{dl}$ en los niños $y$ de $108,5 \pm 26,6 \mathrm{mg} / \mathrm{dl}$ en las niñas y de triglicéridos de $84,0 \pm 41 \mathrm{mg} / \mathrm{dl}$ en niños y $89,4 \pm 39,1$ $\mathrm{mg} / \mathrm{dl}$ en las mujeres. Los promedios de colesterol total y colesterol LDL disminuyeron en ambos sexos a medida que aumentaba la edad, pero manteniendo un valor más alto en las niñas que en los niños. Los triglicéridos fueron mayores en las niñas que en los niños y aumentaron con la edad hasta los 14 años.

En el estudio efectuado en el departamento del Valle, se encontró el promedio más bajo de colesterol total y colesterol LDL en la zona rural de Buenaventura, con un promedio de 164,6 $\pm 35,5$ $\mathrm{mg} / \mathrm{dl} \mathrm{y} 105,2 \pm 32,1 \mathrm{mg} / \mathrm{dl}$, respectivamente. Para triglicéridos, el valor más bajo reportado en este departamento fue de $83,1 \pm 34,2 \mathrm{mg} / \mathrm{dl}$ en la zona urbana de Buenaventura; las otras zonas estudiadas en el Valle presentaron valores más altos de triglicéridos con respecto a la zona urbana de Buenaventura (31), al estudio de Medellín y al de la población que nosotros estudiamos.

Los resultados de colesterol total y colesterol LDL determinados en la población escolar de otros países muestran una tendencia similar con valores más elevados en las niñas y valores más bajos en los niños (15,32-38); en otros estudios se observan valores similares entre niñas y niños, lo cual es contrario a los resultados de los estudios realizados en población escolar colombiana.

Un estudio en población escolar argentina demostró valores de colesterol total de 163,8 mg/ dl en los niños y de $166 \mathrm{mg} / \mathrm{dl}$ en las niñas, valores considerablemente mayores a los de la población del centro-oriente colombiano, pero semejantes a los de Medellín y el Valle; sin embargo, las diferencias entre sexos en la población argentina 
Cuadro 5. Concentraciones de lípidos según práctica de actividad física y preferencia o rechazo por algunos alimentos. Grupo de escolares de cinco departamentos del centro-oriente de Colombia.

\begin{tabular}{|c|c|c|c|c|c|c|c|c|c|c|c|c|c|c|c|c|}
\hline & \multicolumn{4}{|c|}{ Colesterol total } & \multicolumn{4}{|c|}{ Colesterol LDL } & \multicolumn{4}{|c|}{ Colesterol HDL } & \multicolumn{4}{|c|}{ Triglicéridos } \\
\hline & $\begin{array}{c}X \\
\mathrm{mg} / \mathrm{dl}\end{array}$ & DE & LEVENE* & $\begin{array}{c}\text { Pruebat } \\
\text { para la } \\
\text { igualdad de } \\
\text { medias* }^{\star}\end{array}$ & $\begin{array}{c}\mathrm{X} \\
\mathrm{mg} / \mathrm{dl}\end{array}$ & DE & LEVENE ${ }^{*}$ & $\begin{array}{c}\text { Prueba t } \\
\text { para la } \\
\text { igualdad de } \\
\text { medias }^{\star}\end{array}$ & x & DE & LEVENE $^{\star}$ & $\begin{array}{c}\text { Pruebat } \\
\text { para la } \\
\text { igualdad de } \\
\text { medias* }^{\star}\end{array}$ & $x$ & DE & LEVENE* & $\begin{array}{c}\text { Prueba t } \\
\text { para la } \\
\text { igualdad de } \\
\text { medias* }^{*}\end{array}$ \\
\hline \multicolumn{17}{|c|}{ Actividad física } \\
\hline $\begin{array}{l}\text { Realiza actividad } \\
\text { física }\end{array}$ & 149,0 & 28,2 & & & 88,7 & 25,7 & & & 42,3 & 10,1 & & & 89,8 & 38,9 & & \\
\hline No realiza activida & & & 0,98 & 0,70 & & & 0,95 & 0,79 & & & 0,46 & 0,90 & & & 0,47 & 0,48 \\
\hline física & 150,4 & 31,6 & & & 89,6 & 29,0 & & & 42,1 & 9,1 & & & 93,2 & 41,8 & & \\
\hline \multicolumn{17}{|c|}{ Preferencia de consumo alimentos } \\
\hline $\begin{array}{l}\text { Mantequilla - } \\
\text { manteca y aceite } \\
\text { vegetal } \\
\mathrm{N}=463\end{array}$ & & & & & & & & & & & & & & & & \\
\hline $\begin{array}{l}\text { Consume } \\
\mathrm{N}=331\end{array}$ & 152,8 & & 0,22 & 0,21 & 92,6 & 27,1 & 0,56 & 0,18 & 42,9 & & 0,78 & 0,46 & & 36,6 & 0,82 & 0,44 \\
\hline $\begin{array}{l}\text { No consume } \\
\mathrm{N}=132 \\
\text { Comidas rápidas } \\
\mathrm{N}=464\end{array}$ & 148,8 & 30,0 & , & & 88,8 & 26,7 & & & 42,1 & 10,1 & & & 90,0 & 38,6 & & \\
\hline $\begin{array}{l}\text { Consume } \\
\mathrm{N}=432\end{array}$ & 151,5 & & 0,71 & 0,80 & 91,3 & & 0,99 & 0,58 & 42,7 & 10,1 & 0,35 & 0,37 & 87,8 & 37,8 & 0,33 & 0,97 \\
\hline $\begin{array}{l}\text { No consume } \\
\mathrm{N}=32 \\
\text { Azúcar- } \text { miel } \\
\mathrm{N}=\mathbf{4 2 4}\end{array}$ & 152,8 & 26,6 & & & 94,0 & 25,2 & & & 41,2 & 9,0 & & & 88,0 & 28,4 & & \\
\hline $\begin{array}{l}\text { Consume } \\
N=424\end{array}$ & & & 0,01 & 0,88 & & & 0,03 & 0,6 & 42,4 & & 0,63 & 0,10 & 88,1 & 37,7 & 0,29 & 0,61 \\
\hline $\begin{array}{l}\text { No consume } \\
\mathrm{N}=40\end{array}$ & 150,6 & 40,0 & & & 88,5 & 36,8 & & & 45,1 & 9,9 & & & 85,3 & 31,6 & & \\
\hline $\begin{array}{l}\text { Dulces- gaseosas } \\
\mathrm{N}=464\end{array}$ & & & & & & & & & & & & & & & & \\
\hline $\begin{array}{l}\text { Consume } \\
\mathrm{N}=446\end{array}$ & 151,4 & 29,9 & & & 91,4 & 26,9 & & & 42,4 & 9,9 & & & 88,0 & 37,3 & & \\
\hline $\begin{array}{l}\mathrm{N}=446 \\
\mathrm{No} \text { consume } \\
\mathrm{N}=18\end{array}$ & 157,6 & 32,0 & 0,85 & 0,44 & 93,5 & 30,8 & 0,41 & $0, / 8$ & 47,5 & 10,0 & $0, / 6$ & 0,05 & 82,8 & 35,3 & 0,81 & 0,56 \\
\hline
\end{tabular}

* Igualdad de varianzas cuando $p>0,05$

**Prueba $t$ para diferencia de medias suponiendo varianzas desiguales, significativo $p<0,05$

no fueron significativamente diferentes. En colesterol LDL, el valor argentino fue superior en los niños, de $97,1 \mathrm{mg} / \mathrm{dl}$, mientras que en las niñas fue de aproximadamente $95,6 \mathrm{mg} / \mathrm{dl}$, pero $\mathrm{sin}$ diferencias estadísticamente significativas. En triglicéridos los valores argentinos fueron más bajos a los observados en Colombia, con un valor promedio de 70,1 mg/dl en los niños y de 75,9 $\mathrm{mg} / \mathrm{dl}$ en las niñas (33).

Un estudio en escolares venezolanos reveló diferencias en los lípidos también relacionadas con el sexo, con valores de colesterol total, colesterol LDL y triglicéridos más elevados en las niñas de 7-12 años de edad y concentraciones de colesterol HDL más bajas. Los autores del estudio consideran que el aumento en la modernización de la sociedad venezolana es la explicación a la similitud con los valores de lípidos de población pediátrica estadounidense e indican un aumento del riesgo para enfermedad coronaria en la edad adulta (34).

En población escolar brasilera de 7 a 18 años de edad, los valores observados también difieren de los colombianos. La media de colesterol total en población brasilera fue de $162 \pm 28 \mathrm{mg} / \mathrm{dl}$, en triglicéridos de $93 \pm 47 \mathrm{mg} / \mathrm{dl}$, en colesterol HDL de $53 \pm 10 \mathrm{mg} / \mathrm{dl}$ y en colesterol LDL de $92 \pm 24 \mathrm{mg} /$ $\mathrm{dl}$; los hallazgos mostraron que las niñas y los escolares de raza negra tienen los valores más altos de lípidos (35).

Las concentraciones de colesterol observadas en población estadounidense son distintas según el grupo étnico y más altos a los de la población escolar de los cinco departamentos evaluados en 
Cuadro 6. Concentraciones de lípidos según clasificación del índice de masa corporal. Grupo de escolares de cinco departamentos del centro-oriente de Colombia.

\begin{tabular}{|c|c|c|c|c|c|c|c|c|c|}
\hline \multirow[t]{2}{*}{$\begin{array}{l}\text { Clasificiación de índice } \\
\text { de masa corporal }\end{array}$} & \multirow[t]{2}{*}{$\mathbf{N}$} & \multicolumn{2}{|c|}{$\begin{array}{c}\text { Colesterol total } \\
\mathrm{mg} / \mathrm{dl}\end{array}$} & \multicolumn{2}{|c|}{$\begin{array}{c}\text { Colesterol HDL } \\
\text { mg/dl }\end{array}$} & \multicolumn{2}{|c|}{$\begin{array}{c}\text { Colesterol LDL } \\
\text { mg/dl }\end{array}$} & \multicolumn{2}{|c|}{$\begin{array}{c}\text { Triglicéridos } \\
11-12 \\
\mathrm{mg} / \mathrm{dl}\end{array}$} \\
\hline & & $\square$ & $\mathrm{DE}$ & $\square$ & $\mathrm{DE}$ & $\square$ & $\mathrm{DE}$ & $\square$ & $\mathrm{DE}$ \\
\hline Normal & 441 & 151,8 & 29,6 & 43,0 & 10,7 & 92,0 & 26,2 & 83,6 & 37,0 \\
\hline Sobrepeso & 45 & 145,1 & 34,2 & 41,0 & 10,2 & 84,8 & 31,9 & 96,4 & 49,9 \\
\hline Obesidad & 14 & 146,6 & 39,4 & 40,7 & 8,6 & 88,4 & 32,7 & 88,6 & 38,8 \\
\hline Bajo peso & 177 & 147,9 & 27,3 & 43,7 & 10,0 & 88,9 & 25,1 & 76,6 & 30,5 \\
\hline \multirow{2}{*}{$\begin{array}{l}\text { TOTAL } \\
\text { ANOVA } \\
\text { (análisis de varianza de un } \\
\text { factor) significativo } \quad P<0,05\end{array}$} & 677 & & & & & & & & \\
\hline & & \multicolumn{2}{|c|}{0,44} & \multicolumn{2}{|c|}{0,37} & \multicolumn{2}{|c|}{0,36} & \multicolumn{2}{|c|}{$0,00^{*}$} \\
\hline
\end{tabular}

\footnotetext{
* Hay diferencia significativa en las concentraciones de triglicéridos según la clasificación de índice de masa corporal. Por Sheffe la diferencia es entre las concentraciones de triglicéridos de los escolares con déficit de peso y los escolares con sobrepeso $\mathrm{P}<0,05$
}

este estudio. Al comparar los valores de colesterol de población americana con respecto a los otros estudios realizados en población colombiana, siguen siendo elevados pero la diferencia es menor. En población negra, el colesterol total en el rango de edad de 9-11 años es de $173 \mathrm{mg} / \mathrm{dl} \mathrm{y}$ en niños entre 12 y 15 años de 169 mg/dl; en población blanca, es de $170 \mathrm{mg} / \mathrm{dl}$ y $159 \mathrm{mg} / \mathrm{dl}$, respectivamente, y de $168 \mathrm{mg} / \mathrm{dl} \mathrm{y} 160 \mathrm{mg} / \mathrm{dl}$ en población mexicana (36).

En población escolar japonesa se observó un valor promedio de colesterol de $168 \mathrm{mg} / \mathrm{dl}$ entre los $9 \mathrm{y}$ 15 años de edad (37). Este valor parece ser más alto que los valores de colesterol total hallados en población blanca y mexicana de Estados Unidos (36) y a los valores hallados en población colombiana. Los estudios sobre concentraciones de lípidos en escolares de países industrializados como Japón muestran un aumento progresivo en las concentraciones de colesterol, especialmente en las mujeres; estos cambios los atribuyen a la adopción de prácticas occidentales de alimentación, en las cuales el consumo de grasa es mayor (37).

En cuanto a colesterol HDL, los niños de la población estudio presentaron concentraciones más altas de colesterol HDL que las niñas. Al comparar los resultados con los hallados en la ciudad de Medellín y el Valle $(17,31)$, se observa que los valores de colesterol HDL son similares; en Medellín, la concentración promedio en mujeres fue de $44,5 \pm 8,9 \mathrm{mg} / \mathrm{dl}$ y en varones, de $44,2 \pm 9,4$ $\mathrm{mg} / \mathrm{dl}$; los autores encontraron que el promedio de colesterol HDL en las niñas se mantuvo estable, mientras que en los niños disminuyó en el grupo de 15 a 18 años (17). En el Valle, el promedio de colesterol oscilo entre $40,1 \pm 11, \mathrm{mg} /$ dl 3 y $43,9 \pm 9,0 \mathrm{mg} / \mathrm{dl}$ (31).

Al comparar los resultados de colesterol total, colesterol LDL, triglicéridos y colesterol HDL con otros países, se observa que las concentraciones de colesterol total y colesterol LDL tienden a ser inferiores o similares; en los triglicéridos se observa mayor variabilidad, con valores que pueden ser mayores en nuestra población y, para colesterol HDL, los estudios colombianos muestran valores más bajos a los hallados en otros grupos de población $(33,34-37)$.

Este hallazgo sugiere un efecto negativo que podría aumentar el riesgo futuro para enfermedad cardiovascular, teniendo en cuenta que está ampliamente demostrada la asociación inversa entre niveles plasmáticos de colesterol unido a lipoproteínas de alta densidad y ateroesclerosis (38-41); también, indicaría que el riesgo de enfermedad en nuestra población estaría más 
relacionado con las concentraciones de triglicéridos que con el colesterol total o el colesterol LDL.

Se requieren más estudios para indicar cuáles son las variables lipídicas que preponderantemente están más relacionadas con el perfil de morbimortalidad por enfermedad cardiovascular en nuestro país. Promover la actividad física y modificar la alimentación hacia estilos saludables pueden ser algunas de las estrategias para aumentar las concentraciones de colesterol HDL y disminuir los triglicéridos en la población $(34,42)$. A diferencia de nuestros resultados, en algunos estudios en población escolar se ha observado que el sobrepeso se asocia a concentraciones bajas de colesterol HDL (42).

La observación de mayores concentraciones de colesterol HDL en los niños que en las niñas coincide también con lo reportado en otros estudios en población colombiana y de otros países $(16,17,27,34,41,43)$. El mantenimiento de las concentraciones de colesterol HDL, tanto en niñas como en niños en todos los grupos de edad, con una disminución a los 13-15 años pero sin diferencias significativas, concuerda con otras investigaciones en las cuales se menciona que el colesterol HDL se mantiene constante con poca variabilidad alrededor de la adolescencia $(15,16)$.

La tendencia de las concentraciones de lípidos y apolipoproteínas observada en los escolares es contraria a la que se presenta en la población adulta, en la cual las mujeres tienden a tener concentraciones más elevadas de colesterol HDL y ApoA-I, mientras que los hombres tienden a tener concentraciones más altas de colesterol total y colesterol LDL (44).

Se observó que el colesterol total y el colesterol LDL aumentan progresivamente, tanto en niños como en niñas, pero existen diferencias en el periodo de edad en el cual empieza a darse la disminución: en las niñas los valores se redujeron a partir de los 7 años y en los niños, a partir de los nueve años. Lo anterior puede estar asociado al hecho de que las niñas inician su periodo de desarrollo puberal a más temprana edad que los niños y, por tanto, con cambios anticipados a nivel bioquímico (18). La variabilidad también puede tener alguna relación con cambios dietéticos por el inicio de la edad escolar (45-47).

Los triglicéridos presentaron un aumento progresivo desde la niñez hasta la pubertad, con diferencias significativas sólo entre las concentraciones del grupo de niños de 5-6 y de 11-12 años y sin diferencias significativas en los diferentes grupos de edad de las niñas. Por otro lado, se observó que en el grupo de 13-15 años las concentraciones en todas las variables lipídicas disminuían. Estos efectos pueden estar relacionados con el proceso de maduración sexual, más evidente en el rango de 13 a 15 años $(6,8,9,11)$, con cambios en la composición corporal entre los escolares según la edad y el sexo, y con factores ambientales, como las diferencias que pueden existir en los hábitos de alimentación y la actividad física (48-50).

Estudios en población escolar latinoamericana explican que las concentraciones de lípidos disminuyen del rango considerado de alerta al aumentar la edad, lo que pudiera explicarse en parte por cambios en la dieta y la actividad física (51). En el caso de nuestra población, el aumento de triglicéridos en los niños de mayor edad con diferencias significativas con respecto a los de menor edad pudiera estar asociado, en parte, a cambios desfavorables en la alimentación y a disminución de la actividad física.

El no hallar diferencias significativas entre las concentraciones de lípidos de la población que realiza actividad física y la población que no realiza actividad física, así como entre los que prefieren alimentos grasos o ricos en carbohidratos simples (cuadro 5), sugiere variaciones en las variables lipídicas por factores de edad, sexo y proceso de maduración sexual, y no de factores ambientales como alimentación y nivel de actividad física. Este resultado concuerda con los estudios que determinan mayor influencia de las variables biológicas en los niños (3-10), especialmente para colesterol total y colesterol LDL (2). No obstante, pueden existir sesgos con respecto a los resultados obtenidos, porque en el estudio se consultó sobre actividad física, considerando práctica deportiva vigorosa, en la cual existen cambios de composición corporal y 
bioquímico, o práctica de actividad física, en la cual se incrementa el gasto energético, pero no necesariamente puede llegar a generar cambios notables en la composición del individuo o en sus variables bioquímicas (52-54).

Igualmente, la evaluación sobre alimentación de los escolares debe realizarse con un método más exacto para determinar si existen diferencias en las concentraciones de los lípidos por efecto de la alimentación. Es probable que, como se ha observado en otros estudios (2), la concentración de triglicéridos en nuestro grupo de población dependa mayoritariamente del efecto asociado a la alimentación o al nivel de actividad física, y no a los factores metabólicos y genéticos. Esto explicaría por qué los valores más bajos de triglicéridos se observaron en los escolares con déficit de peso y no en los que tenían sobrepeso.

La justificación para no encontrar relación entre índice de masa corporal y las demás variables lipídicas podría ser que este indicador de dimensión corporal no es el más sensible para determinar la relación con los lípidos; existen investigaciones en las cuales se determina que el peso y el índice de masa corporal son factores adecuados de predicción de las concentraciones plasmáticas de lípidos; sin embargo, las medidas de pliegue tricipital están más fuertemente asociadas con las concentraciones de lípidos en ambos sexos (12).

Las concentraciones de Apo A-I y B-100 no presentaron diferencias estadísticamente significativas según el grupo de edad, pero se observó que en el rango de 13-15 años las concentraciones se inclinaban a valores más bajos, debido tal vez al proceso de maduración sexual. Los resultados mostraron algunas diferencias por sexo en las concentraciones de ApoB-100 y ApoA-I: las niñas tenían mayores concentraciones de ApoB-100 y menores concentraciones de ApoA-I que los niños. Estas variaciones en la ApoB-100 en las niñas se relacionan con los hallazgos de mayores concentraciones de colesterol total y colesterol LDL y menores de colesterol HDL en ellas (19).

Los resultados para ApoA-I muestran diferencias con un estudio en niños argentinos, donde se encontró un valor de $146 \mathrm{mg} / \mathrm{dl}$ para la ApoA-I en los varones del grupo de 6-10 años, el cual fue significativamente más alto que el valor de 124 $\mathrm{mg} / \mathrm{dl}(\mathrm{p}<0,05)$ del grupo de varones de 11-13 años. En las niñas se observó una media de Apo A-I de $138 \mathrm{mg} / \mathrm{dl}$ para el grupo de 6-10 años, valor mayor al de $117 \mathrm{mg} / \mathrm{dl}(\mathrm{p}<0,05)$ del grupo de 11-13 años. A diferencia de la ApoA-I, la tendencia a no mostrar diferencias significativas en las concentraciones de ApoB-100 dentro del mismo sexo para los grupos de edad también se observó en los escolares argentinos.

En forma similar a lo hallado en otras investigaciones, las concentraciones de apolipoproteínas en los niños de este estudio muestran que los varones tienen un perfil lipoproteico menos aterogénico que las niñas en los rangos de edad estudiados (32).

No fue posible examinar los resultados sobre valores de lípidos en percentiles según sexo y grupo de edad con respecto a los obtenidos en otros estudios (55), porque los rangos de edad y los percentiles que se definieron en cada estudio difieren; no obstante, es importante anexarlos como referencia para observar las tendencias en las concentraciones de los lípidos en una población escolar colombiana.

En conclusión, se encontraron variaciones en los lípidos explicadas por los cambios de edad y sexo; posiblemente las variaciones están determinadas por factores adicionales, pero se requieren otras investigaciones para establecer la contribución de cada variable. La promoción de estilos de vida saludable puede contribuir a reducir el riesgo futuro de enfermedad cardiovascular relacionado con bajas concentraciones de colesterol HDL y mayores de triglicéridos en esta población.

\section{Agradecimientos}

Agradecemos a las instituciones de salud y educación departamentales y municipales que facilitaron y apoyaron el trabajo de campo para la recolección de la información, a los padres y niños participantes por su participación y paciencia, a Colciencias entidad cofinanciadora del proyecto y a Carlos Corredor, Director del Programa de 
Postgrados, Facultad de Ciencias, Pontificia Universidad Javeriana, por su asesoría en la elaboración del artículo.

\section{Conflicto de intereses}

Los autores manifiestan que no existen conflictos de intereses en la elaboración y ejecución de este proyecto.

\section{Financiación}

Instituto Nacional de Salud, Pontificia Universidad Javeriana y Colciencias proyecto código 2104-0411811.

\section{Referencias}

1. Casanueva V, Milos C, Lopetegui B, Chiang MT, Cid $\mathbf{X}$. Influencia de la etnia y el ambiente sobre el perfil lipídico en escolares de la VIII región. Rev Méd Chile. 1994;122:496-502.

2. Chen TJ, Ji CY, Pang ZC, Wang SJ, Hu YH, Qin Y. Heritability of serum lipids and lipoproteins and its related factors in twins aged 5 to 19 years of China. Zhonghua Yu Fang Yi Xue Za Zhi. 2004;38:237-9.

3. Bellu R, Ortisi MT, Scaglioni S, Agostoni C, Salanitri VS, Riva E, et al. Lipid and apoprotein A-I and B levels in obese school-age children: results of a study in the Milan area. J Pediatr Gastroenterol Nutr. 1993; 16:446-50.

4. González-Requejo A, Sánchez-Bayle M, Baeza J, Arnaiz P, Vila S, Asensio J, et al. Relations between nutrient intake and serum lipid and apolipoprotein levels. J Pediatr. 1995;127:53-7.

5. Frerichs RR, Webber LS, Srinivasan SR, Berenson GS. Relation of serum lipids and lipoproteins to obesity and sexual maturity in white and black children. Am J Epidemiol. 1978;108:486-96.

6. Berenson GS, Foster TA, Frank GC, Frerichs RR, Srinivasan SR, Voors AW, et al. Cardiovascular disease risk factor variables at the preschool age. The Bogalusa heart study. Circulation. 1978;57:603-12.

7. Berenson GS, Blonde CV, Farris RP, Foster TA, Frank GC, Srinivasan SR, et al. Cardiovascular disease risk factor variables during the first year of life. Am J Dis Child. 1979;133:1049-57.

8. Frerichs RR, Srinivasan SR, Webber LS, Berenson GR. Serum cholesterol and triglyceride levels in 3446 children from a biracial community: The Bogalusa Heart Study. Circulation. 1976;54:302-9.

9. Berenson GS, Srinivasan SR, Cresanta JL, Foster TA, Webber LS. Dynamic changes of serum lipoproteins in children during adolescence and sexual maturation. Am J Epidemiol. 1981;113:157-70.
10. Rainwater DL, Mitchell BD, Comuzzie AG, Haffner SM. Relationship of low-density lipoprotein particle size and measures of adiposity. Int $\mathrm{J}$ Obes Relat Metab Disord. 1999;23:180-9.

11. Rodríguez C, Pablos-Méndez A, Palmas W, Lantigua R, Mayeux R, Berglund L. Comparison of modifiable determinants of lipids and lipoprotein levels among African-Americans, Hispanics, and Non-Hispanic Caucasians $>$ or $=65$ years of age living in New York City. Am J Cardiol. 2002;89:178-83.

12. Chu NF, Rimm EB, Wang DJ, Liou HS, Shieh SM. Relationship between anthropometric variables and lipid levels among school children: The Taipei Children Heart Study. Int J Obes Relat Metab Disord. 1998;22:66-72.

13. Rask-Nissila L, Jokinen E, Ronnemaa T, Viikari J, Tammi A, Niinikoski $\mathrm{H}$, et al. Prospective, randomized, infancy-onset trial of the effects of a low-saturated-fat, low-cholesterol diet on serum lipids and lipoproteins before school age: The Special Turku Coronary Risk Factor Intervention Project (STRIP). Circulation. 2000;102:1477-83

14. Chueca S, Nasarre M, García L, Aznar M, Almarza L, Sánchez B. Lipoprotein profile determinants in children and adolescents from a lipid consultation clinic. The impact of diet, body composition and physical activity. An Esp Pediatr. 1997;47:357-62.

15. Reilly SL, Kottke BA, Sing Cl. The effects of generation and gender on the joint distributions of lipid and apolipoprotein phenotypes in the population at large. $\mathrm{J}$ Clin Epidemiol. 1990;43:921-40.

16. Webber LS, Srinivasan SR, Wattigney WA, Berenson GS. Tracking of serum lipids and lipoproteins from childhood to aduthood. Am J Epidemiol .1991;133:884-99.

17. Uscátegui Peñuela RM, Álvarez Uribe MC, Laguado Salinas I, Soler Terranova W, Martínez Maluendas $\mathrm{L}$, Arias Arteaga $\mathbf{R}$, et al. Factores de riesgo cardiovascular en niños de 6 a 18 años de Medellín (Colombia). An Esp Pediatr. 2003;58:411-7.

18. Eisenberg S, Levy RI. Lipoprotein metabolism. Adv Lipid Res. 1975;13:1-89.

19. Kim HJ, Kalkhoff RK. Changes in lipoprotein composition during the menstrual cycle. Metabolism. 1979;28:663-8.

20. Bachorik PS, Lovejoy KL, Carroll MD, Johnson CL. Apolipoprotein B and A-I distributions in the United State 1988-1991: Results of the national health and nutrition examination survey III (NHANES III). Clin Chem. 1997;43:2364-78.

21. Brotons C, Ribera A, Perich RM, Abrodos D, Magana P, Pablo S, et al. Worldwide distribution of blood lipids and lipoproteins in childhood and adolescence: a review study. Atherosclerosis. 1998;139:1-9

22. Lucas D, Greaves D. Atheroesclerosis: role of chemokines and macrophages. Exp Rev Med. 2001;3:1-18. 
23. Ministerio de Salud, Instituto Nacional de Salud, Organización Panamericana de Salud. Situación de salud en Colombia. Indicadores básicos. Bogotá D.C.: Ministerio de Salud, Instituto Nacional de Salud, Organización Panamericana de Salud; 2002.

24. González M, De la Hoz F. Mortalidad por enfermedades crónicas no transmisibles en Colombia, 1990-1999. Inf Quinc Epidemiol Nac. 2002;14:209-36.

25. Martínez González J, Llorente Cortés V, Badimon L. Biología celular y molecular de las lesiones ateroescleróticas. Rev Esp Cardiol. 2001;54:218-31.

26. Berenson GS, Srinivasan SR, Bao W, Newman WP 3rd, Tracy RE, Wattigney WA. Association between multiple cardiovascular risk factors and atherosclerosis in children and young adults. $\mathrm{N}$ Engl $\mathrm{J}$ Med. 1998;338:1650-6.

27. Rosner B, Prineas R, Loggie J, Daniels SR. Percentiles for body mass index in U.S. children 5 to 17 years of age. J Pediatr. 1998;132:211-22.

28. Must A, Dallal GE, Dietz WH. Reference data for obesity: 85th and 95th percentiles of body mass index (wt/ ht2) and triceps skinfold thickness. Am J Clin Nutr. 1991;53:839-46.

29. Hernández-Valera Y, Henríquez PG, Arenas 0 , García Blanco M, Cardena Y. Índice de masa corporal P/T2. Valores para diagnóstico de desnutrición en niños venezolanos de 2 a 10 años de edad. XXV Jornadas Nac Ped Puer 1986. En: Manual de crecimiento y desarrollo. Caracas: FUNDACREDESA; 1997.p.186.

30. Friedewald WT, Levy RI, Fredrickson DS. Estimation of the concentration of low density lipoprotein cholesterol in plasma, without use of the separative ultracentrifuge. Clin Chem. 1972;18:499-502.

31. Gracia B, de Plata C, Pradilla A, Leiva J. Factores de riesgo para enfermedades de mayor prevalencia en el Valle del Cauca útiles para el desarrollo de estrategias de prevención. Colombia Médica. 2003;34:47-55.

32. Freedman DS, Srinivasan SR, Harsha DW, Webber LS, Berenson GS. Relation of body fat patterning to lipid and lipoprotein concentrations in children and adolescents: The Bogalusa Heart Study. Am J Clin Nutr. 1989;50:930-9.

33. Morales MT, Sánchez M, Peláez M, Puente MJ, Ruiz C, Antón AJ. Valores del perfil lipídico y de los índices CT/C-HDL, C-LDL/C-HDL, Apo B/A e índice aterogénico, en niños de 6 años de Rivas-Vaciamadrid. An Esp Pediatr. 1998;49:140-4.

34. Aguilar D, Yánez C, Bina MR, Dilenque S, Peterson G, Tavella M. Lípidos y apolipoproteínas A-I y B séricos en escolares adventistas de la República Argentina. Acta Bioquim Clin Latinoam. 2003;37:5-12.

35. Mendoza S, Contreras G, , Fernández M, Nucete $\mathbf{H}$, Morrison JA, et al. Lipids and lipoproteins in Venezu- elan and American schoolchildren: within and crosscultural comparison. Pediatr Res. 1980;14:272-7.

36. Giuliano IC, Coutinho MS, Freitas SF, Pires MM, Zunino JN, Ribeiro RQ. Serum lipids in school kids and adolescents from Florianópolis, SC, Brazil-Healthy Floripa 2040 study. Arq Bras Cardiol. 2005;85:85-91.

37. Hickman TB, Briefel RR, Carroll MD, Rifkind BM, Cleeman JI, Maurer KR, et al. Distributions and trends of serum lipid levels among United States children and adolescents ages 4-19 years: data from the third national health and nutrition examination survey. Prev Med. 1998;27:879-90.

38. Takasaki Y. Serum lipids levels and factors affecting atherogenic index in Japanese children. J Physiol Anthropol Appl Human Sci. 2005;24:511-5.

39. Rosillo I, Pituelli N, Corbera M, Lioi S, Turco M, Arrigo $\mathbf{M}$, et al. Perfil lipídico en niños y adolescentes de una población escolar. Arch Argent Pediatr. 2005;103:293-7

40. Tall AR. Plasma high density lipoproteins: metabolism and relationship to atherogenesis. J Clin Invest. 1990;86:379-84.

41. Castelli WP, Doyle JT, Gordon T, Hames CG, Hjortland MC, Hulley SB, et al. HDL cholesterol and other lipids in coronary heart disease. The cooperative lipoprotein phenotyping study. Circulation. 1989;55:76772.

42. Gordon D, Basil R. High density lipoprotein. The clinical implications of recent studies. N Engl J Med. 1997;19:1311-5

43. Miller NE, Thelle DS, Forde OH, Mjos OD. The Tromso heart study high density lipoprotein and coronary heart disease: a prospective case control study. Lancet. 1997;1:965-8.

44. Grillo L, Crispim S, Siebert A, Wippel A, Rossi A, de Campos I. Lipid profile and obesity in low income school children. Rev Bras Epidemiol. 2005;8:75-81.

45. Bercedo A, González P, Muñoz P, Albajar M, Rodriguez J, Braga S, et al. Asociación entre el perfil lípidico y genotipo de la apoproteína $E$ en niños españoles (8-15 años). An Esp Pediatr. 1998;49:120-4.

46. Sociedad Española de Arteriosclerosis, Sociedad Española de Medicina Interna y Liga de la Lucha contra la Hipertensión Arterial. Recomendaciones para la prevención primaria de la enfermedad cardiovascular. Clin Invest Arterioscl. 1994;6:62-102.

47. Pose A. ¿Qué significa factor de riesgo cardiovascular? Med Clin (Barc). 1993;101:58-9.

48. Utermann G. Apolipoproteins, quantitative lipoprotein traits and multifactorial hyperlipidaemia. Ciba Found Symp. 1987;130:52-69.

49. Rask-Nissila L, Jokinen E, Viikari J, Tammi A, Rönnemaa T, Marniemi J, et al. Impact of dietary 
intervention, sex, and apolipoprotein $\mathrm{E}$ phenotype on tracking of serum lipids and apolipoproteins in 1-to 5year-old children. The special Turku Coronary Risk Factor Intervention Project (STRIP). Arterioscler Thromb Vasc Biol. 2002;22:492-8.

50. D’Angelo CP, Agüero A, Ghioldi M, Saco M, García R, Lorenzo J. Evaluación morfofuncional, psicosocial y de hábitos alimentarios de los alumnos de las escuelas públicas de la Ciudad de Buenos Aires. Rev Asoc Med Argent. 2005;118:9-22.

51. McMurry MP, Cerqueira MT, Connor SL, Connor WE. Changes in lipid and lipoprotein levels and body weight in Tarahumara Indians after consumption of an affluent diet. N Engl J Med. 1991;325:1704-8.

52. Molero-Conejo E, Morales LM, Fernández V, Raleigh X, Gómez ME, Semprún-Fereira $M$, et al. Lean adolescents with increased risk for metabolic syndrome. Arch Latinoam Nutr. 2003;53:39-46.
53. Yoshioka M, Doucet E, St-Pierre S, Alméras N, Richard D, Labrie A, et al. Impact of high-intensity exercise on energy expenditure, lipid oxidation and body fatness. Int J Obes Relat Metab Disord. 2001;25,332-9.

54. Dionne I, Almeras N, Bouchard C, Tremblay A. The association between vigorous physical activities and fat deposition in male adolescents. Med Sci Sports Exerc. 2000;32:392-5.

55. Seki M, Niyama F, Seki M, Pereira J, Seki M, Bonametti A, et al. Perfil lipídico: intervalos de referência em escolares de 2 a 9 anos de idade da cidade de Maracaí (SP) / Lipid profile: normal range values in school children from two to nine years old from the city of Maracaí, SP, Brazil. J Bras Patol Med Lab. 2003;39:131-7. 